\title{
Caracterización del Patrón de Comportamiento de las Partículas en Suspensión en el Valle de México
}

\author{
Carlos Vázquez ${ }^{(1)}$ Fernando Beristain $^{(2) *}$ y Estela Audelo ${ }^{(3)}$ \\ Universidad Nacional Autónoma de México, Facultad de Estudios Superiores Cuautitlán, \\ (1) Centro de Cómputo, (2) Depto. de Ingeniería y Tecnología (3) Depto. de Química \\ Av. $1^{\circ}$ de mayo S/N Colonia Santa María las Torres, C.P. 54740, Cuautitlán Izcalli, Edo. de \\ México-México. (e-mail: carlos@unam.mx; ferber@unam.mx; audelovu@unam.mx ) \\ ${ }^{*}$ Autor a quien debe ser dirigida la correspondencia
}

Recibido Jul. 15, 2011; Aceptado Ago. 22, 2011; Versión final recibida Ene. 24, 2012

\section{Resumen}

Se estudiaron los registros de la contaminación por partículas en suspensión, con diámetro menor a $10 \mu \mathrm{m}$ (PM10 y concentración mayor a $60 \mu \mathrm{g} / \mathrm{m}^{3}$ (límite de la buena calidad del aire) en tres estaciones de monitoreo en el Valle de México. El estudio comprende los meses de febrero, mayo, agosto y noviembre. Se manejó información por día, fines de semana y días de pago quincenales, para establecer el comportamiento del contaminante. Se encontró que las estaciones del año y el uso de suelo tienen una influencia significativa en los niveles de PM10, distinguiéndose tres horarios que presentan máximos de contaminación. La estación de monitoreo ambiental Merced es la más contaminada, el jueves y el viernes son los días más contaminados de la semana y cuando el día de pago se encuentra entre semana el número de eventos de contaminación aumenta. Los índices de la dinámica no lineal y los gráficos en el espacio de fases indican un comportamiento caótico determinista.

\section{Characterization of the Behavioral Pattern of Suspended Particles in the Valley of Mexico}

\begin{abstract}
The records of pollution by particles in suspension with diameter smaller than $10 \mu \mathrm{m}$ (PM10) with concentration greater than $60 \mathrm{\mu g} / \mathrm{m}^{3}$ (the limit of good quality air) of three monitoring stations in the Metropolitan Zone of Mexico Valley were studied. The study includes the months of February, May, August and November. The study considered information by day, weekends and biweekly pay day, to establish the behavior of the polluting agents It was found that the seasons and the land use have significant influence on the PM10 levels; distinguishing three peaks of contamination events. The Merced station was the most polluted; Thursdays and Fridays are the most contaminated days of the week, and when the biweekly pay day is within the week, the number of contamination events increases. The indices of the nonlinear dynamics and the graphs in the phase space indicate a determinist chaotic behavior.
\end{abstract}




\section{INTRODUCCIÓN}

La Zona Metropolitana del Valle de México (ZMVM), una de las más densamente pobladas en el mundo, se encuentra a $2240 \mathrm{~m}$ sobre el nivel del mar, a esta altitud, la cantidad de oxigeno se encuentra disminuida, lo que hace menos eficiente la combustión; asimismo, rodeada por cadenas montañosas y la alta concentración de fuentes de emisión inhiben la dispersión de los contaminantes (Jazcilevich, 2003); de acuerdo con la red automática de monitoreo ambiental en la ZMVM, los contaminantes críticos del aire son el ozono y el material particulado (RAMA, 2011). En un estudio realizado en México, Ramirez-Rembao et al. (2009) encontraron que los contaminantes ozono, monóxido de carbono, PM10 y los factores climáticos temperatura y humedad, mostraron diferente relación con las infecciones respiratorias agudas. También en México, se desarrolló un modelo de pronóstico de la concentración de ozono usando una red neuronal artificial alimentada con 6 variables meteorológicas y tres químicas, el modelo obtenido tiene una eficiencia global del 50\% (García et al., 2008). En Chile, Celis et al. (2007) en un estudio de la contaminación urbana, concluyeron, que el aumento de la contaminación por PM10 durante otoño e invierno debe ser considerado de origen antropogénico. Las condiciones climáticas, la distribución de contaminantes en el aire y su concentración varían mes a mes, con precipitaciones que ocurren principalmente entre mayo y octubre y escasean de noviembre a mayo (Aymoz et al., 2007); además, la estación del año y las características del uso de suelo afectan la permanencia de las partículas en suspensión (Zakey et al., 2008).

Entre los constituyentes de la atmósfera, el material particulado con diámetro aerodinámico menor o igual a $10 \mu \mathrm{m}$ (PM10), es la fracción respirable de las partículas suspendidas totales (PST) que puede penetrar el tejido pulmonar humano y causar problemas respiratorios, exacerbar enfermedades cardiovasculares y aumentar la incidencia en enfermedades autoinmunes (Diez Roux et al., 2008; Karar et al., 2006; Zeka et al., 2006). Asimismo, Préndez et al. (2007) en un estudio sobre emisiones provenientes de distintas fuentes, concluyeron que el daño a la salud depende de la composición química de las partículas. Las PM10 son de naturaleza compleja ya que son el resultado, no solamente de la emisión directa de partículas sino también de ciertos gases que se condensan directamente en forma de partículas o sufren transformación química (Pérez-Vidal, et al., 2010; Sardar et al., 2005). También, las partículas en suspensión han sido estudiadas como sistema dinámico, reportando que el sistema es sensible a las condiciones iniciales (Vázquez, 2009).

El presente trabajo tiene como objetivo efectuar un estudio descriptivo del comportamiento de las PM10 cuya concentración sea mayor a $60 \mu \mathrm{g} / \mathrm{m}^{3}$, límite inferior en la clasificación de la buena calidad del aire en la ZMVM, estableciendo la relación entre el número de eventos de mala calidad del aire, por la presencia del contaminante, con los arreglos temporales (día, fin de semana, día de pago), la estación del año y las estaciones de monitoreo ambiental, para contrastar la actividad antropomórfica y el uso del suelo con los eventos de mala calidad del aire, aplicando los métodos estadísticos y los métodos de la dinámica no lineal, que permiten establecer esta diferencia y definir los cambios en el comportamiento dinámico del sistema.

\section{MATERIALES Y MÉTODOS}

Se obtuvieron las bases de datos de PM10, del Sistema de Monitoreo Atmosférico de la Ciudad de México (SIMAT), seleccionando los valores cuya concentración fue mayor a $60 \mu \mathrm{g} / \mathrm{m}^{3}$, considerada como el límite entre la buena y mala calidad del aire (SIMAT, 2010). Los registros corresponden a los valores de concentración horaria obtenidos en tres estaciones de monitoreo ambiental distribuidas en la ZMVM: Cerro de la Estrella (CES), Merced (MER) y Pedregal (PED), las cuales se encuentran ubicadas en zonas que difieren fundamentalmente por sus características de densidad de población, tipo de urbanización y uso del suelo (ver tablas 1 y 2 ).

El estudio comprende a febrero, mayo, agosto y noviembre, meses centrales de una división estacionalmente aproximada, que corresponden al invierno, primavera, verano y otoño, en el hemisferio norte. Los datos se dividieron en arreglos por día de la semana, fines de semana 
(viernes a domingo) y día de pago (considerando tres días tomando como central el día de pago). Se definió como evento a las lecturas horarias cuya concentración de PM10 es mayor de 60 $\mu \mathrm{g} / \mathrm{m}^{3}$. Se calcularon los índices de la estadística descriptiva y los índices de la dinámica no lineal para definir una diferencia significativa en los arreglos y caracterizar el comportamiento del contaminante.

Tabla 1: Características del uso de suelo en las tres delegaciones estudiadas

\begin{tabular}{|l|c|c|c|}
\hline \multicolumn{1}{|c|}{ Uso de suelo (\%) } & $\begin{array}{c}\text { Cerro de la } \\
\text { Estrella }\end{array}$ & Merced & Pedregal \\
\hline Habitacional & 58.1 & 37 & 72.3 \\
\hline Mixto (habitación, oficinas, industria y servicios) & 28.7 & 25 & 5.4 \\
\hline Equipamiento (servicios) & 4.3 & 30 & 5.8 \\
\hline Industrial & 3 & 2 & 1.3 \\
\hline Áreas verdes y espacios abiertos & 5.9 & 6 & 15.2 \\
\hline
\end{tabular}

Tabla 2: Principales parámetros en las tres delegaciones estudiadas

\begin{tabular}{|l|c|c|c|}
\hline \multicolumn{1}{|c|}{ Parámetros } & Cerro de la Estrella & Merced & Pedregal \\
\hline Población & $1,821,000$ & 448,000 & 707,000 \\
\hline Superficie $\left(\mathrm{Km}^{2}\right)$ & 116.13 & 33.42 & 96.17 \\
\hline Densidad & 15,681 & 13,405 & 7,352 \\
\hline Flujo vehicular & Alto & Alto & Bajo \\
\hline Temperatura anual media $\left({ }^{\circ} \mathrm{C}\right)$ & 16.6 & 16.0 & 16 \\
\hline Precipitación anual media $(\mathrm{mm})$ & 616.8 & 600 & 1000 \\
\hline
\end{tabular}

\section{RESULTADOS Y DISCUSIÓN}

En la tabla 3 se muestra el porcentaje de eventos que exceden la norma de buena calidad del aire, se observa que febrero es el que presenta el mayor porcentaje y agosto el menor, en todas las estaciones de monitoreo. Los eventos que exceden el límite de la buena calidad del aire están relacionados con la estación del año, así durante el invierno (febrero), el valle de México conjuga la presencia de tolvaneras con las inversiones térmicas propiciando un aumento en el número de eventos de contaminación, de la misma manera la temporada de lluvias propicia una menor contaminación en el verano (agosto).

Tabla 3: Porcentaje de eventos de mala calidad del aire

\begin{tabular}{|c|c|c|c|c|c|c|c|c|c|c|c|}
\hline \multicolumn{4}{|c|}{ Merced } & \multicolumn{4}{c|}{ Cerro de la Estrella } & \multicolumn{5}{c|}{ Pedregal } \\
\hline Feb & May & Ago & Nov & Feb & May & Ago & Nov & Feb & May & Ago & Nov \\
\hline 59.9 & 20.7 & 4.83 & 25.0 & 49 & 18.7 & 8.3 & 22.7 & 29.3 & 8.5 & 4.4 & 12.2 \\
\hline
\end{tabular}

Para encontrar los horarios de mayor contaminación, se dividieron los datos en 3 turnos: matutino de 0 a $12 \mathrm{~h}$; vespertino de 13 a $18 \mathrm{~h}$ y nocturno de 19 a $24 \mathrm{~h}$. En forma general, en las tres estaciones de monitoreo y las 4 estaciones del año, en el turno matutino el pico de mayor contaminación se encuentra de 9 a $10 \mathrm{~h}$; en el turno vespertino de 15 a $16 \mathrm{~h}$ y en el nocturno de 19 a $20 \mathrm{~h}$, estos corresponden a periodos en los que se presenta el mayor tránsito de personas y vehículos, correspondiente al inicio y fin de las actividades de escuelas, oficinas y empresas, relacionadas también con el horario de comida. En la figura 1 se muestran las lecturas horarias de la concentración de PM10 los cuatro lunes del mes de febrero en las tres estaciones de monitoreo. 


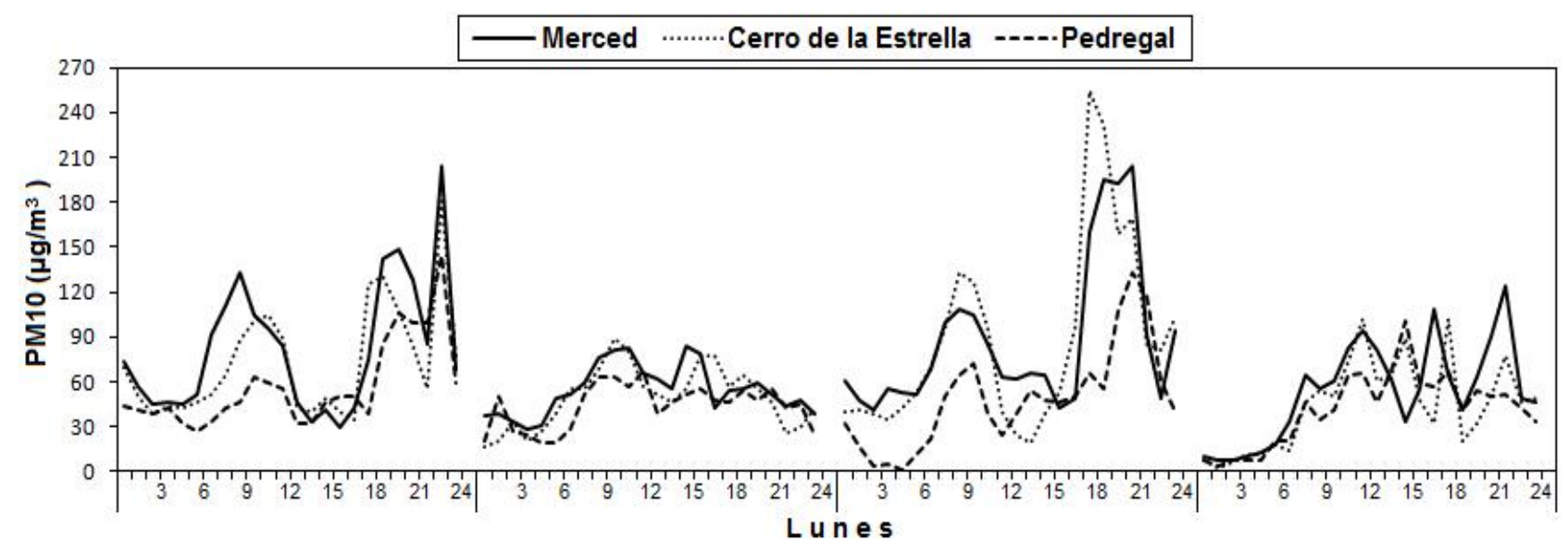

Fig. 1: Horarios de mayor contaminación

Los datos representados en la figura 2, son datos puntuales que se obtuvieron de sumar el número de eventos de cada día de la semana por mes; donde se muestra que los días con mayor número de eventos de contaminación son el jueves y el viernes, se considera que esto es por la acumulación de la concentración de PM10 a lo largo de la semana y el aumento del flujo vehicular previo a las ventas de fin de semana. Analizando los meses estudiados, agosto se distingue por tener la menor cantidad de eventos y febrero la mayor. La Merced es la estación de monitoreo con más eventos de contaminación, seguida por Cerro de la Estrella, mientras que la estación Pedregal es la menos contaminada.

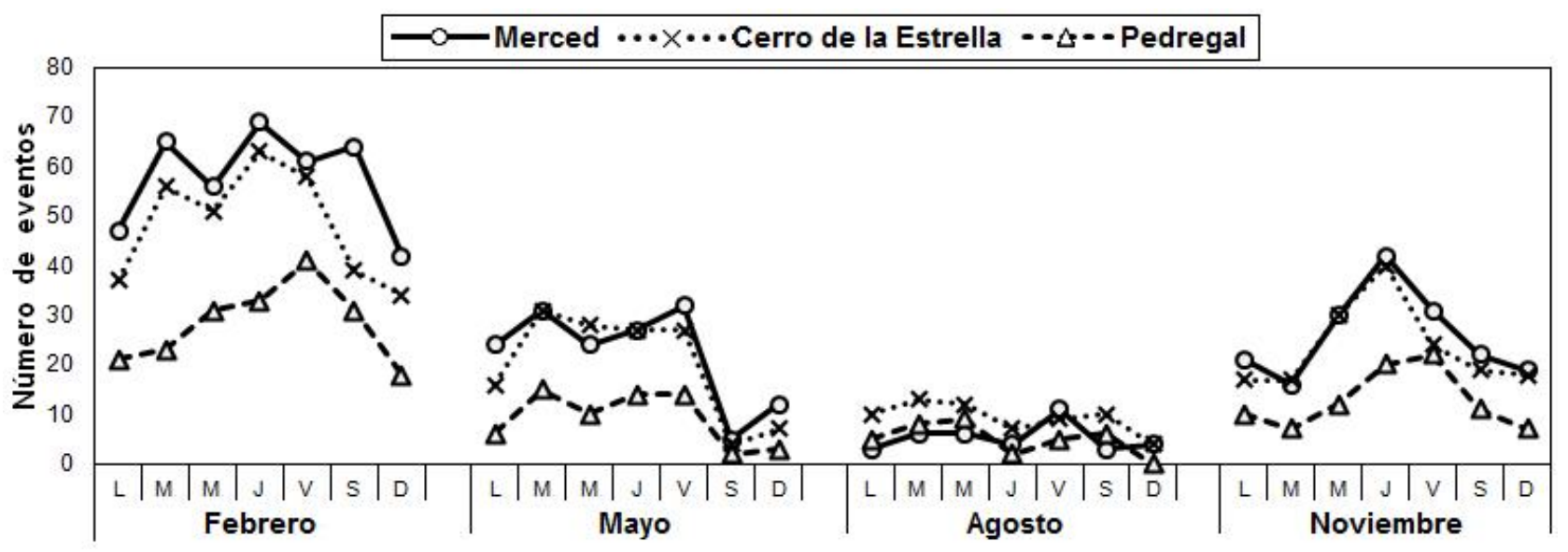

Fig. 2: Número de eventos mayores a $60 \mu \mathrm{g} / \mathrm{m}^{3}$ por día de la semana

Para el análisis de los días de pago, que en México corresponden a los días quince y último de cada mes, el presente estudio toma los días 14, 15 y 16 llamando a este arreglo quincena 1 (Q1) y los días 29, 30 y 31 llamando a este arreglo quincena 2 (Q2). En la tabla 4 se muestra el número de eventos que se presentan por quincena, por mes y por estación de monitoreo; estos resultados explican el comportamiento del contaminante señalando al mes de febrero y la estación de monitoreo Merced con el mayor número de eventos.

El uso de suelo en las estaciones de monitoreo ambiental estudiadas, establece las diferencias en los grados de contaminación mostrados; las zonas donde se encuentran Merced y Cerro de la Estrella son similares en cuanto al tipo de flujo vehicular (transporte público y de carga), densidad

poblacional y áreas verdes, lo que las diferencia, es que la zona de ubicación de la estación Merced tiene un porcentaje cinco veces mayor de uso de suelo para servicios y el comercio en ésta es de menudeo, mientras que en Cerro de la Estrella es de mayoreo; esto ocasiona que la Merced presente más eventos de mala calidad. El contraste con la estación Pedregal, que es la 
que presenta menos eventos, es su alta proporción de espacios verdes, el porcentaje de uso de suelo habitacional es mayor y el flujo vehicular, caracterizado por vehículos particulares, es bajo.

Tabla 4: Número de eventos mayores de $60 \mu \mathrm{g} / \mathrm{m}^{3}$

\begin{tabular}{|c|c|c|c|c|c|c|c|c|}
\hline \multirow{2}{*}{$\begin{array}{c}\text { Estación de } \\
\text { monitoreo }\end{array}$} & \multicolumn{2}{|c|}{ Febrero } & \multicolumn{2}{|c|}{ Mayo } & \multicolumn{2}{c|}{ Agosto } & \multicolumn{2}{c|}{ Noviembre } \\
\cline { 2 - 10 } & Q1 & Q2 & Q1 & Q2 & Q1 & Q2 & Q1 & Q2 \\
\hline MER & 57 & 41 & 7 & 9 & 8 & 1 & 9 & 16 \\
\hline CES & 48 & 24 & 6 & 13 & 7 & 4 & 14 & 22 \\
\hline PED & 28 & 18 & 2 & 3 & 8 & 4 & 6 & 12 \\
\hline
\end{tabular}

En los diagramas de cajas y bigotes, que representan los datos de PM10 cuya concentración es mayor de $60 \mu \mathrm{g} / \mathrm{m}^{3}$, se observa en la figura 3, una asimetría positiva, lo que nos indica una mayor dispersión de los datos de mayor concentración; los días de pago en los meses de febrero y noviembre presentan un mayor número de datos atípicos por una probable influencia de la temporada seca en el valle de México y el predominio del invierno. Estacionalmente el mes que presenta mayor variabilidad es febrero, asimismo, al compararse mes a mes, se presenta una diferencia significativa en el comportamiento del contaminante.

Comparando los días de pago con los fines de semana se observa que los fines de semana de febrero representan el mayor problema de contaminación, presentando un número considerable de valores atípicos que llegan por arriba de $250 \mu \mathrm{g} / \mathrm{m} 3$, mientras que los días de pago, muestran datos que, de manera normal, llegan hasta los $150 \mu \mathrm{g} / \mathrm{m} 3$ (ver figura 3).

a) Análisis por día de pago

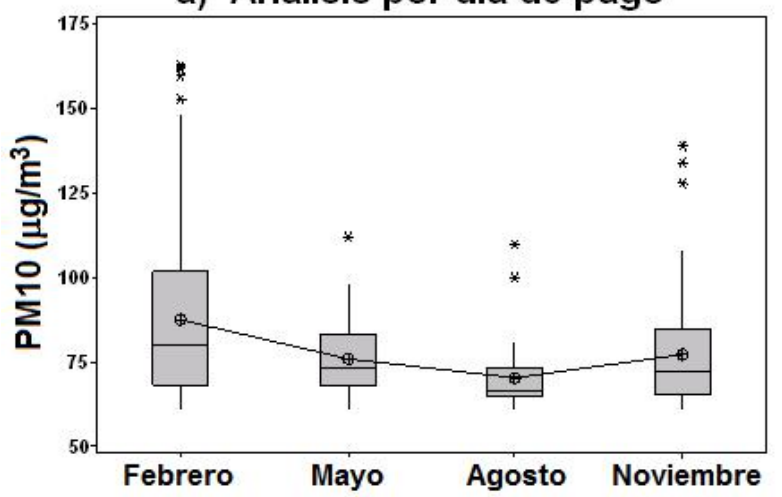

b) Análisis por fin de semana

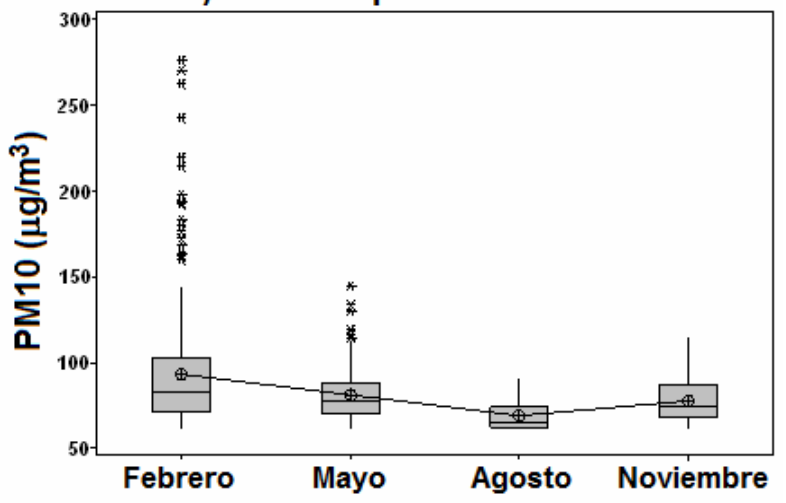

Fig. 3: Diagrama de caja y bigotes en el análisis por día de pago y fin de semana

Los índices de la estadística descriptiva, muestra que los días de pago de febrero y noviembre, son los de mayor dispersión, corroborado por la varianza; el valor de la probabilidad muestra que existe una diferencia significativa en el comportamiento de la contaminación entre las estaciones de monitoreo en el mes de febrero, fundamentalmente con la estación Pedregal, y que de la misma manera esta diferencia se marca entre las estaciones del año, contrastados nuevamente por el mes de febrero (representante del invierno). Los fines de semana de febrero, la estación Pedregal se diferencia de la Merced y Cerro de la Estrella (con una alta varianza) presentando una menor dispersión de los datos de la contaminación, lo mismo sucede en el mes de noviembre, aunque la variabilidad de los datos de las estaciones Merced y Cerro de la Estrella son menores que en el mes de febrero; nuevamente los fines de semana de febrero (relacionado con el invierno) son los que presentan una mayor variabilidad. La tabla 5 muestra la diferencia, por fin de semana y día de pago, por cada estación de monitoreo y por estación del año. 
Tabla 5: Comparación del día de pago y fin de semana

\begin{tabular}{|c|c|c|c|c|c|c|c|c|c|c|c|c|c|}
\hline & \multirow{2}{*}{ Estadísticos } & \multicolumn{3}{|c|}{ Febrero } & \multicolumn{3}{|c|}{ Mayo } & \multicolumn{3}{|c|}{ Agosto } & \multicolumn{3}{|c|}{ Noviembre } \\
\hline & & CES & MER & PED & CES & MER & PED & CES & MER & PED & CES & MER & PED \\
\hline \multirow{4}{*}{$\begin{array}{l}\text { Día de } \\
\text { pago }\end{array}$} & Media & 85.9 & 93.2 & 77.7 & 79.9 & 75.9 & 79.0 & 70.8 & 75.1 & 66 & 78.3 & 79.7 & 70.7 \\
\hline & $\begin{array}{l}\text { Desviación } \\
\text { estándar }\end{array}$ & 22.3 & 26.1 & 16.4 & 8.01 & 13.6 & 14.3 & 10.3 & 14.3 & 4.2 & 16.4 & 19.9 & 8.8 \\
\hline & Varianza & 500 & 683 & 271 & 64 & 185 & 203 & 105 & 205 & 18 & 267 & 399 & 76 \\
\hline & Probabilidad & \multicolumn{3}{|c|}{0.002} & \multicolumn{3}{|c|}{0.787} & \multicolumn{3}{|c|}{0.175} & \multicolumn{3}{|c|}{0.176} \\
\hline \multirow{4}{*}{$\begin{array}{l}\text { Fin de } \\
\text { semana }\end{array}$} & Media & 95.7 & 94.3 & 80.3 & 82.4 & 78.3 & 74.2 & 69.8 & 71.4 & 63.7 & 79.8 & 78.9 & 71.3 \\
\hline & $\begin{array}{l}\text { Desviación } \\
\text { estándar }\end{array}$ & 34.5 & 33.2 & 15.9 & 17.8 & 11.0 & 8.9 & 9.3 & 8.6 & 3.8 & 14.1 & 12.8 & 7.5 \\
\hline & Varianza & 1192 & 1103 & 251 & 317 & 121 & 78 & 85 & 74 & 14 & 197 & 164 & 56 \\
\hline & Probabilidad & \multicolumn{3}{|c|}{0.00} & \multicolumn{3}{|c|}{102} & \multicolumn{3}{|c|}{0.08} & \multicolumn{3}{|c|}{0.002} \\
\hline
\end{tabular}

Los resultados en cuanto a los índices de la dinámica no lineal no muestran diferencias significativas en ningún arreglo temporal. Los valores del exponente de Hurst, todos menores de 0.5 , son indicativos de un comportamiento antipersistente. La entropía de Kolmogorov con valores de entre 0.4 y 0.6 revela una pérdida de información. El exponente de Lyapunov, cuyos valores oscilan entre 0.4 y 0.6 indica que el sistema es divergente con sensibilidad a las condiciones iniciales. La dimensión de correlación cuyos valores oscilan entre 3.6 y 5.6 son indicativos de un sistema con una complejidad baja. En la tabla 6 se muestran los índices de los arreglos de fin de semana.

Tabla 6: Índices de la estación de monitoreo Merced

\begin{tabular}{|l|c|c|c|c|}
\hline & $\begin{array}{c}\text { Exponente de } \\
\text { Lyapunov }\end{array}$ & $\begin{array}{c}\text { Dimensión de } \\
\text { Correlación }\end{array}$ & $\begin{array}{c}\text { Exponente de } \\
\text { Hurst }\end{array}$ & $\begin{array}{c}\text { Entropía de } \\
\text { Kolmogorov }\end{array}$ \\
\hline Febrero & $0.473 \pm 0.116$ & $4.318 \pm 0.613$ & 0.195 & 0.694 \\
\hline Mayo & $0.556 \pm 0.098$ & $4.407 \pm 0.337$ & 0.150 & 0.482 \\
\hline Agosto & $0.567 \pm 0.107$ & $4.213 \pm 0.479$ & 0.242 & 0.447 \\
\hline Noviembre & $0.600 \pm 0.084$ & $3.611 \pm 0.280$ & 0.254 & 0.470 \\
\hline
\end{tabular}

Analizando los gráficos en el espacio fase para los días de la semana se observa que los atractores tiene una estructura similar para los días de lunes a sábado, los cuales forman una figura cónica; los domingos el atractor no presenta ninguna estructura, en todas las estaciones y meses. En el análisis por quincena no se aprecia una estructura definida, esto debido a que la cantidad de puntos es mínima ya que solamente se están considerando en cada arreglo los eventos de tres días del mes. Para el caso de los arreglos de fin de semana y del mes, los gráficos presentan una estructura semejante para todas las estaciones de monitoreo, mostrando atractores que tienden a una estructura cónica, con una densidad de puntos mayor del inicio hasta las tres cuartas partes del atractor con puntos que salen de la estructura general. En la figura 4 se muestran los atractores del arreglo de cada mes de la estación de monitoreo Merced, observándose que en los meses de febrero y mayo las escalas de los ejes son mas grandes con respecto a los otros meses, esto es, las magnitudes de los puntos es mayor.

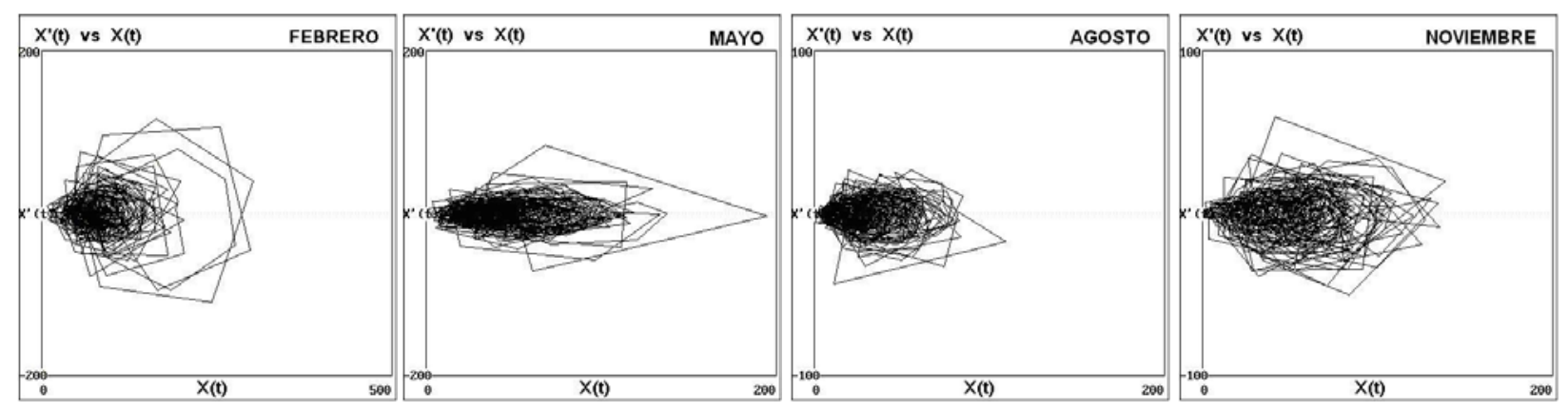

Fig. 4: Atractores de cada mes en la estación de monitoreo Merced 


\section{CONCLUSIONES}

De acuerdo a los estudios realizados, febrero, que estacionalmente corresponde al invierno, tiene el más alto nivel de horas que exceden a la norma y la mayor variabilidad y dispersión en la concentración del contaminante. Corresponde a los días jueves y viernes y a la quincena 1 el mayor número de eventos en las tres estaciones de monitoreo. Se encontraron tres picos de contaminación en los horarios de 9 a 10, 15 a 16 y 19 a 20 horas. La estación de monitoreo Merced es la más contaminada.

Los índices de la dinámica no lineal y los gráficos en el espacio de fases indican un comportamiento caótico determinista, manteniendo baja la medida de la predicción en todos los arreglos temporales.

Los resultados encontrados muestran que el comportamiento de las PM10 está influenciado, por el uso de suelo y la actividad antropogénica, pero es la estación del año la que exacerba los problemas de la contaminación.

Las probables soluciones a la contaminación por PM10 serian: la implementación de un programa de transporte escolar obligatorio, reforzar los programas de restricción de circulación para el transporte altamente contaminante, la reforestación de las áreas dañadas y un control estricto para evitar la invasión de zonas verdes y de reserva ecológica con unidades habitacionales.

\section{REFERENCIAS}

Aymoz, G., Jaffrezo, J.L., Chapuis, D., Cozic, J. y Maenhaut, W., Seasonal variation of PM10 main constituents in two valleys of the French Alps. I: EC/OC fractions, Atmos. Chem. Phys. 7, 661-675 (2007).

Celis, J.E., Morales, J.R., Zaror, C.A. y Carvacho, O.F., Contaminación del Aire Atmosférico por Material Particulado en una Ciudad Intermedia: El caso de Chillán (Chile), Información Tecnológica 18(3), 49-58 (2007).

Diez Roux, A.V., Auchincloss, A.H., Franklin, T.G., Raghunathan, T., Graham Barr, R., Kaufman, J., Astor, B. y Keeler, J., Long-term Exposure to Ambient Particulate Matter and Prevalence of Subclinical Atherosclerosis in the Multi-Ethnic Study of Atherosclerosis, American Journal of Epidemiology 167 (6), 667-675 DOI: 10.1093/aje/kwm359 (2008).

García, I., Marbán, A., Tenorio, Y.M. y Rodríguez, J.G., Pronóstico de la Concentración de Ozono en Guadalajara-México usando Redes Neuronales Artificiales, Información Tecnológica 19(3), 8996 (2008).

Jazcilevich, A.D., García, A.R. y Ruíz-Suárez, L.G., A study of air flow patterns affecting pollutant concentrations in the Central Region of Mexico, Atmospheric Environment 37 (2), 183-193 (2003).

Karar, K., Gupta, A.K., Kumar A. y Biswas, A.K., Seasonal variations of PM10 and TSP in residential and Industrial sites in an urban area of Kolkata, India, Environmental Monitoring and Assessment 118, 369-381 (2006).

Pérez-Vidal, H., Lunagómez-Rocha M.A. y Acosta-Pérez L.I., Análisis de Partículas Suspendidas Totales (PST) y Partículas Fracción Respirable (PM10), en Cunduacán Tabasco, Uciencia 26(2), 151-162 (2010).

Préndez , M., Corvalán R.M. y Cisternas M., Estudio Preliminar del Material Particulado de Fuentes Estacionarias: Aplicación al Sistema de Compensación de Emisiones en la Región Metropolitana, Chile, Información Tecnológica 18(2), 93-103 (2007). 
RAMA, http://www.sma.df.gob.mx/simat2/informaciontecnica (enero 2011).

Ramírez-Rembao, M., Rojas, R.I. y Garcia-Cueto R., Influencia de los Contaminantes Atmosféricos en las Infecciones Respiratorias Agudas en Mexicali-Baja California, México, Información Tecnológica 20(3), 89-100 (2009).

Sardar, S.B., Fine, P.M. y Sioutas, C., Seasonal and spatial variability of the size-resolved chemical composition of particulate matter (PM10) in the Los Angeles Basin, Journal of Geophysical Research 110, D07S08, doi:10.1029/2004JD004627, (2005).

SIMAT, http://www.calidadaire.df.gob.mx/calidadaire (enero 2010).

Vázquez-Cruz, C., Estudio del Comportamiento dinámico de la contaminación por material particulado PM10 en la zona metropolitana del Valle de México, Tesis de Maestría, Facultad de Ingeniería, Universidad Nacional Autónoma de México, México (2009).

Zakey, A.S., Abdel-Wahab, M.M., Petterson, J.B.C., Gatari, M.J. y Hallquist, M., Seasonal and spatial variation of atmospheric particulate matter in a developing megacity, the Greater Cairo, Egypt, Atmósfera 21(2), 171-189 (2008).

Zeka, A., Zanobetti, A. y Schwartz, J., Individual-Level Modifiers of the Effects of Particulate Matter on Daily Mortality, American Journal of Epidemiology 163 (9), 849-859 (2006). 